\title{
Biopelículas activas de extracto acuosos de Gliricidia sepium y su influencia en la vida útil microbiológica del queso costeño
}

\section{Active biofilms of aqueous extract of Gliricidia sepium and its influence on the microbiological shelf life of coastal cheese}

\author{
Rafael González-Cuello; Fidel Guardo-Palomino²; Somaris Quintana-Martínez ${ }^{3}$
}

\begin{abstract}
${ }^{1}$ Microbiólogo, M.Sc., Ph.D. Universidad de Cartagena, Facultad de Ingeniería, Programa Ingeniería de Alimentos, Grupo de Investigación en Ingeniería de Fluidos Complejos y Reología de Alimentos (IFCRA). Cartagena - Bolívar, Colombia; e-mail: rgonzalezc1@unicartagena.edu.co; (D) https://orcid.org/00000002-2674-2876

${ }^{2}$ Ing. de Alimentos. Universidad de Cartagena, Facultad de Ingeniería, Programa Ingeniería de Alimentos, Grupo de Investigación en Ingeniería de Fluidos Complejos y Reología de Alimentos (IFCRA). Cartagena - Bolívar, Colombia; e-mail: fguardop@unicartagena.edu.co; (D) https://orcid.org/0000-0002-18548598

${ }^{3}$ M.Sc. Universidad de Cartagena, Facultad de Ingeniería, Programa Ingeniería de Alimentos, Grupo de Investigación en Ingeniería de Fluidos Complejos y Reología de Alimentos (IFCRA). Cartagena - Bolívar, Colombia; e-mail: squintanam@unicartagena.edu.co; (D) https://orcid.org/0000-0003-4716-0354
\end{abstract}

Cómo citar: González-Cuello, R.; Guardo-Palomino, F.; Quintana-Martínez, S. 2021. Biopelículas activas de extracto acuosos de Gliricidia sepium y su influencia en la vida útil microbiológica del queso costeño. Rev. U.D.C.A Act. \& Div. Cient. $24(1): e 1467$. http://doi.org/10.31910/rudca.v24.n1.2021.1467

Artículo de acceso abierto publicado por Revista U.D.C.A Actualidad \& Divulgación Científica, bajo una licencia Creative Commons CC BY-NC 4.0

Publicación oficial de la Universidad de Ciencias Aplicadas y Ambientales U.D.C.A, Institución de Educación Superior Acreditada de Alta Calidad por el Ministerio de Educación Nacional.

Recibido: febrero 10 de $2020 \quad$ Aceptado: enero 27 de 2021

Editado por: Rita María Ávila G. de Hernández

\section{RESUMEN}

La elaboración de biopelículas con propiedades bioactivas es un área interesante en el campo de los empaques alimentarios. El objetivo de este estudio fue obtener biopelículas activas (BPA) a base de extracto acuoso de hojas de Gliricidia sepium y determinar su efecto en la vida útil microbiológica del queso costeño. Para la fabricación de las BPA, el extracto acuoso fue microencapsulado, mediante gelación iónica y, posteriormente, incorporado en las biopelículas. La determinación de la vida útil de muestras de queso costeño, se llevó a cabo mediante microbiología predictiva, utilizando el modelo de Monod Hinshelwood. Las microcápsulas utilizadas tuvieron un diámetro promedio de $273,786 \mu \mathrm{m}$. Los resultados mostraron un aumento en la vida útil microbiológica de 26,7 días, en quesos con $\mathrm{BPA}$, almacenado a $7^{\circ} \mathrm{C}$, en comparación con una muestra control (sin BPA), confirmando que las BPA investigadas ejercen un efecto inhibitorio sobre los microorganismos, causantes de deterioro en quesos. Por tal motivo, la metodología aquí planteada puede ser una alternativa en la conservación de un producto perecedero, como el queso costeño.

Palabras clave: Biopolímeros; Microencapsulación; Gelación iónica; Productos lácteos.

\section{ABSTRACT}

The elaboration of biofilms with bioactive properties is an interesting area in the field of food packaging. The aim of this study was to obtain active biofilms (AB) based on aqueous extract of Gliricidia sepium leaves and determine their effect on the microbiological 
shelf life of coastal cheese. For the manufacture of the AB, the aqueous extract was microencapsulated by means of ionic gelation and later incorporated in the biofilms. The coastal cheese's shelf life was carried out by means of predictive microbiology using the Monod Hinshelwood model. The microcapsules had an average diameter of $273.786 \mu \mathrm{m}$. The results showed an increase in the microbiological shelf life of 26.7 days in cheeses with $\mathrm{AB}$ stored at $7^{\circ} \mathrm{C}$ compared with control sample (without $\mathrm{AB}$ ) confirming that the $\mathrm{AB}$ investigated exerts an inhibitory effect on the microorganisms causing deterioration in cheeses. For this reason, the methodology proposed here can be an alternative in the conservation of a perishable product such as coastal cheese.

Keywords: Biopolymers; Microencapsulation; Ionic gelation; Dairy products.

\section{INTRODUCCIÓN}

Uno de los grandes intereses por parte de la industria alimenticia es la prolongación de la vida útil de los alimentos, sin comprometer su estructura, conservando las características propias, como el color, el aroma, el sabor y la textura, así como sus componentes nutricionales. Las biopelículas (BP), se presentan como una interesante alternativa para la conservación de los alimentos y solución frente a la problemática medio ambiental que polímeros sintéticos causan, debido a su prolongado periodo de degradación y toxicidad (González et al. 2016).

Existe una variedad de materiales empleados para el diseño de BP; entre los principales, se encuentran proteínas, lípidos, polisacáridos y sus mezclas (Costa et al. 2018). Dentro de los polisacáridos, el carragenato es uno de los más utilizados para el diseño de BP. Es un biopolímero lineal aniónico soluble en agua, obtenido a partir de algas rojas, que promueven la formación de geles, a través de un proceso de secado moderado, en el que se evapora el disolvente, formando una red tridimensional, a partir de la liberación de dobles hélices, formando una película sólida con capacidad de retención de agua y una estructura superficial homogénea (Gie et al. 2018; Osorio et al. 2017).

Las biopelículas activas (BPA) son un segmento interesante en el área de empaques, que consiste en la adición de compuestos activos con propiedades antimicrobianas y antioxidantes (Arnon \& Poverenov, 2018). Estos compuestos activos pueden ser extraídos de plantas, como el matarratón (Gliricidia sepium), un árbol caducifolio, perteneciente a la familia de las leguminosas, nativo de Meso América, Centroamérica y del norte de Sudamérica, que logra alcanzar alturas de hasta 12 metros (Patil et al. 2015). Dentro de su composición, se ha reportado la presencia de pinitol, cumarinas, hidrocarburos, flavonas, isoflavonas, pterocarpanos y flavonoides glucosídicos, en hojas y raíces (Kabore et al. 2012; Von Son-de Fernex et al. 2017). Entre sus aplicaciones, se encuentran propiedades bioactivas, como antioxidante, antimicrobiano e insecticidas (Patil et al 2015; Ríos- De Álvarez et al. 2012; Sinha, 2013).
La desventaja de las BPA, se presenta al momento de su elaboración, en el que parte del compuesto activo se pierde producto de la evaporación, limitando, de esta forma, la acción antimicrobiana de la BPA (Peralta et al. 2015), por lo que se plantea proteger el principio activo, mediante la microencapsulación, dado que esta técnica permite retenerlo dentro de una membrana fuerte, semipermeable y esférica, con diámetros que van desde micras hasta $1 \mathrm{~mm}$ (Cano et al. 2016; Castro-Rosas et al. 2017). De esta forma, el principio activo, se contiene por más tiempo, evitando las pérdidas por procesamiento, para luego liberarse de forma gradual y controlada en un medio definido, mediante tecnologías emergentes, como el ultrasonido.

El ultrasonido (US) es el nombre que reciben las ondas sonoras con frecuencias mayores a aquellas que pueden ser detectadas por el oído humano. Es una forma de energía que viaja en ondas de sonido iguales o mayores a 20.000 vibraciones por segundo (Hoover, 2000; Bermúdez-Aguirre \& Barbosa-Cánovas, 2008; Rezek et al. 2008; Arroyo et al. 2011). El US ha sido utilizado ampliamente en procesos de extracción de compuestos, a partir de material vegetal (De Sousa et al. 2020). El principal efecto del US en los procesos de extracción es el incremento de la permeabilidad del material, que contiene el compuesto a extraer. A medida que la temperatura aumenta, se forman burbujas de cavitación, que colapsan y aumentan la presión, conllevando a una ruptura del material, facilitando la penetración del disolvente en la matriz, por tanto, hay un mayor contacto del disolvente con el analito, promoviendo una mayor solubilidad (Huang et al. 2019).

Por otra parte, el queso costeño es un producto autóctono de la Costa Caribe Colombiana, región que se caracteriza por una alta producción de leche bovina (aproximadamente, el 60\% de la producción total del país). Se caracteriza por ser una variedad de queso no madurado, es decir, fresco, elaborado con leche de vaca sin pasteurizar, con un sabor salado entre moderado y fuerte (Ruíz et al. 2017). Al tratarse de un producto con alto porcentaje en producción artesanal con técnicas rudimentarias, en las que predominan las malas condiciones higiénico sanitarias en su procesamiento, se convierte en un producto alimenticio con alto contenido de microrganismos patógenos, como Salmonella, L. monocytogenes y S. aureus, causantes de intoxicación alimentaria (Frazier et al. 2003; Ruíz et al. 2017).

Por lo mencionado anteriormente, el objetivo del presente trabajo fue evaluar la influencia del ultrasonido en el desarrollo de BPA, a base de matarratón (Gliricidia sepium) y su incidencia en la vida útil microbiológica del queso costeño.

\section{MATERIALES Y MÉTODOS}

Obtención de extracto acuoso. Se recolectaron hojas verdes de G. sepium en el cerro de la popa, ubicado en la ciudad de Cartagena, a $148 \mathrm{~m}$ s.n.m., en árboles de edad madura (14 a 20 años). Se realizó una clasificación, selección, lavado y desinfección de las hojas, en una solución de hipoclorito, a 200ppm, durante $5 \mathrm{~min}$. Posteriormente, fueron secadas por 4 días, a $32^{\circ} \mathrm{C}$. La extracción del principio activo, se llevó a cabo mediante hidrodestilación por arrastre con vapor, 
siguiendo la metodología propuesta por González et al. (2018), utilizando un sistema de destilación compuesto.

Microencapsulación. Las microcápsulas fueron elaboradas utilizando metodología propuesta por González et al. (2013), usando dispersiones poliméricas de Goma Gelana de bajo acilo $(0,75 \% \mathrm{p} / \mathrm{v})$ (Modernist Panty Kitchen Alchemy) y carbonato de calcio (30mM) en agua, bajo agitación constante $(300 \mathrm{rpm})$, a $90^{\circ} \mathrm{C}$, por $15 \mathrm{~min}$. Luego, se dejó enfriar la preparación, con el fin de incorporar el EA $(40 \% \mathrm{v} / \mathrm{v})$ y evitar así la volatilización de los compuestos presentes en el extracto. Posteriormente, el proceso de gelificación, se realizó mediante la incorporación de $\delta$-gluconolactona hasta alcanzar un pH de 4,2. A la dispersión polimérica, se le agregó aceite de canola en una proporción ( $1: 2 \mathrm{v} / \mathrm{v})$ (dispersión: aceite), bajo agitación constante por $15 \mathrm{~min}$, aumentado las revoluciones, formando una emulsión. Finalmente, el aceite es removido por adsorción y las microcápsulas contenidas en la fase acuosa fueron centrifugadas (Hamilton Bell VanGuard V6000) dos veces, a 5000rpm, durante $10 \mathrm{~min}$, con solución salina $(0,85 \% \mathrm{NaCl})$ y almacenadas a $4^{\circ} \mathrm{C}$, hasta su uso.

Determinación del diámetro de las microcápsulas. $100 \mu \mathrm{L}$ de microcápsulas fue utilizado para determinar el diámetro de las microcápsulas. La muestra fue diluida en una solución salina estéril en un portaobjetos y analizado en un microscopio óptico (Carl Zeiss Microscopy GmbH, Kongsalee 9 - 21), integrado a una cámara digital. Las microfotografías obtenidas fueron procesadas con ayuda del programa de cómputo Zen Image Pro-Plus versión 4.

Obtención de biopelículas. Las BPA fueron realizadas por método de Casting, siguiendo la metodología propuesta por
González et al. (2018), empleando Kappa Carrageenan 0,35\% p/v, como biopolímero y carbonato de Calcio $\left(\mathrm{CaCO}_{3}\right) 30 \mathrm{mM}$, como donador de cationes. Los reactivos fueron disueltos en agua destilada conteniendo glicerol al 12\% v/v (plastificante). Posteriormente, la mezcla fue sometida a calentamiento bajo agitación constante, por un periodo de $15 \mathrm{~min}$, a $90^{\circ} \mathrm{C}$. Una vez transcurrido el tiempo, se dejó enfriar hasta $40^{\circ} \mathrm{C}$ y se adicionaron las microcápsulas $(100 \mu \mathrm{L})$ y $\delta$-Gluconolactona, con el fin de liberar los cationes presentes en el carbonato y facilitar la gelificación del polímero. Para la formación de las BPA, se adicionaron volúmenes específicos (10mL) de cada solución en cajas de Petri. Luego, se realizó el secado en una incubadora (memmert IN 160), a $40^{\circ} \mathrm{C}$, durante 72 horas, aproximadamente, asegurando la uniformidad de las BPA.

Aplicación de ultrasonido. Las BPA con área conocida fueron sometidas a tratamiento ultrasónico $40 \mathrm{KHz}, 40^{\circ} \mathrm{C}$, por $10 \mathrm{~min}$, en un baño ultrasónico (Labware Scientific Model KSL5120-5). Esta relación de potencia temperatura y tiempo seleccionada, se basó en estudios cinéticos de liberación principios activos, llevados a cabo en el grupo de investigación.

Estimación de la vida útil microbiológica. Para determinar la funcionalidad de las BPA sobre el queso costeño, se llevó a cabo la estimación de la vida útil microbiológica de esta matriz alimentaria. Tres cinéticas de crecimiento a distintas temperaturas $\left(7-37^{\circ} \mathrm{C}\right)$ fueron construidas por triplicado, reportando el promedio aritmético de los 15 puntos utilizados. Las bacterias aerobias mesófilas fueron usadas como microorganismos indicadores. Es importante destacar que los datos de las cinéticas se modelaron matemáticamente, mediante la ecuación de Baranyi \& Roberts (1994) (Ecuación 1), por medio del programa de cómputo DMFit 2.0:

$$
y(t)=y_{0}+\mu_{\max } t+\frac{1}{\mu_{\max }} \ln \left(e^{-v t}+e^{-h_{0}}-e^{-v t-h_{0}}\right)-\frac{1}{m} \ln \left[1+\frac{e^{m \mu_{\max } t+\frac{1}{\mu_{\max }} \ln \left(e^{-v t}+e^{-h_{0}}-e^{\left.-v t-h_{0}\right)}\right.}-1}{e^{m\left(y \max -y_{0}\right)}}\right]
$$

ecuación 1

Donde, $y(t)$ es la concentración celular o el diámetro de las colonias, yo es la concentración o diámetro inicial, umáx es la velocidad específica de crecimiento $(1 / \mathrm{h})$, m es un parámetro de curvatura para caracterizar la transición de la fase exponencial, $\mathbf{v}$ es un parámetro de curvatura para caracterizar la transición a la fase exponencial y ho es un parámetro adimensional que cuantifica el estado fisiológico inicial de las células. Una vez obtenidos los parámetros cinéticos de crecimiento microbiano, se procedió a estimar la vida útil microbiológica utilizando la ecuación de Monod-Hinshelwood (Ecuación 2) (González et al. 2016).

$$
\text { ts }=\frac{\log N_{s}-\log N_{0}}{\log 2} \mathrm{xT}_{\mathrm{d}}
$$

ecuación 2

Donde, ts es el tiempo necesario para que se desarrolle la alteración en el alimento, $\mathrm{N}_{\mathrm{s}}(\mathrm{ufc} / \mathrm{g})$ es el valor correspondiente a la población de seguridad (valor máximo permisible antes de considerarse alterado el producto), No (ufc/g) es el valor correspondiente a la población inicial presente en el producto y $\mathrm{T}_{\mathrm{d}}$ es el tiempo duplicación de la población alterante específica.

\section{RESULTADOS Y DISCUSIÓN}

Diámetro de las microcápsulas. La microscopía óptica reveló la forma de las microcápsulas obtenidas con gelana de bajo acilo. En la figura 1, se aprecian estructuras esféricas rígidas bien definidas después de los procesos de centrifugación, que tienen lugar en la etapa de separación de las microcápsulas; este tipo de estructuras son originadas por la reacción de los iones divalentes con los grupos carboxilo de las moléculas de gelana (Chandrasekaran \& Thailambal, 1990; Sanderson, 1990).

En cuanto a los diámetros de las microcápsulas, se obtuvieron valores promedios de $273,768 \pm 0,564 \mu \mathrm{m}$ y un área promedio de $60685,893 \mu^{2}$. Es importante resaltar que estos valores fueron 


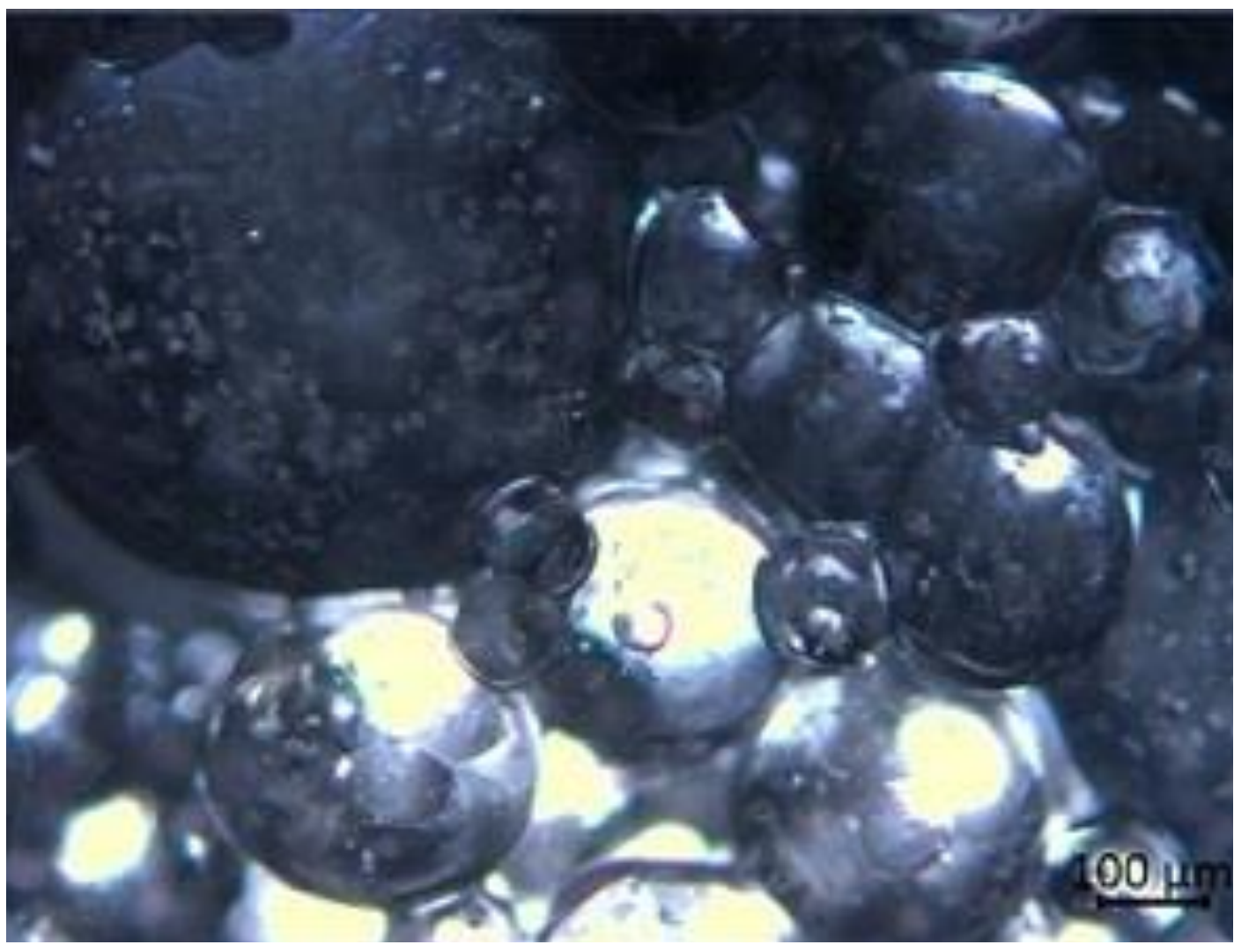

Figura 1. Micrografías de microcápsulas con aumento de 100X.

calculados a partir de cinco repeticiones por microfotografía. El tamaño de las microcápsulas es un aspecto relevante cuando son incorporadas a matrices alimentarias, ya que el tamaño puede afectar las características sensoriales de los productos alimenticios (Tyle, 1993). Además, la adición de microcápsulas en biopelículas conlleva a una modificación de las propiedades mecánicas y reológicas de las mismas, como fue previamente reportado por González et al. (2018).

Microcápsulas con diámetros grandes ofrecen un mayor porcentaje de encapsulación y protección del compuesto activo, pero una pobre dispersión en el medio; por otro lado, diámetros muy pequeños, dan como resultado microcápsulas huecas y con baja eficiencia de encapsulación (Zhao et al. 2008). Un diámetro óptimo para las microencapsulaciones, se sitúa entre un máximo de $1 \mathrm{~mm}$ y un mínimo de 80um (Cano et al. 2016; Castro-Rosas et al. 2017).

Estimación de vida útil microbiológica. Este parámetro, se puede determinar mediante la estimación de los parámetros cinéticos de crecimiento microbiano del microorganismo indicador, previo modelado de los datos, utilizando la ecuación de Baranyi \& Roberts (1994). Los valores obtenidos en la estimación de la vida útil microbiológica, se presentan en la tabla 1 , donde se puede apreciar que, el mayor tiempo de vida útil (42,8 días), se encontró en muestras de queso recubiertas con la BPA, mientras que las muestras de queso sin BPA mostraron una vida útil de 16,1 días. Este resultado puede ser atribuido a la presencia de compuestos con características antimicrobianas presentes en el extracto acuoso de G. sepium. Es notable destacar que la bioactividad (antioxidante, antimicrobiana, antifungica e insecticida) de esta legumbre ha sido reportada previamente (Nazli et al. 2011; Sinha, 2013). En la figura 2 , se observa la velocidad de cinética de crecimiento microbiano en queso costeño en un rango de temperaturas de 7 a $37^{\circ} \mathrm{C}$, demostrando que la aplicación de la BPA es capaz de incrementar el tiempo de duplicación en las temperaturas evaluadas, conllevando al mencionado aumento en la vida útil microbiológica, debido a una disminución en los tiempos de duplicación celular.

De acuerdo con la resolución 02310 de 1986, capítulo XVIII, artículo 129 del Ministerio de Salud de la República de Colombia, el queso fresco refrigerado en empaque hermético tiene una duración sanitaria aproximada de 18 días y refrigerado empacado al vacío, de 30 días. Considerando los resultados obtenidos en la presente investigación, la aplicación de BPA, a base de goma gelana y conteniendo microcápsulas cagadas con extracto acuoso, logra incrementar la vida útil del queso 26,7 días, aproximadamente. Estos resultados son similares a los publicados por Gorrasi et al. (2016), quienes reportaron un incremento de 20 días en la vida útil microbiológica en queso mozarela, empacados en un recubrimiento activo, diseñado a partir de tereftalato de polietileno (PET) e hidróxido doble en capas (LDH), intercalado con aniones de salicilato y carbonato dispersos en un sellado térmico, a base de solvente.

En términos generales, la aplicación de BPA conteniendo extracto acuoso de G. sepium logra incrementar la vida útil microbiológica 


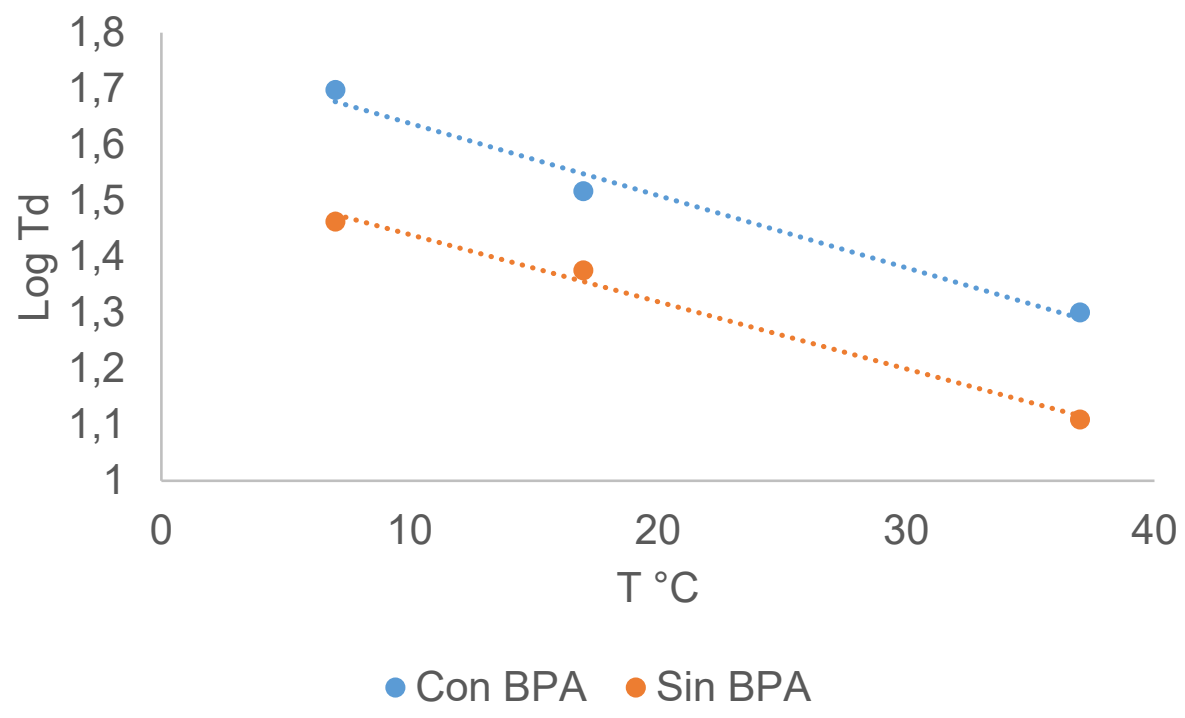

Figura 2. Velocidad de cinética de crecimiento microbiano en queso costeño a diferentes temperaturas, sin biopelícula y con biopelícula activa.

Tabla 1. Valores de estimación de vida útil en queso costeño, sin biopelícula y con biopelícula activa.

\begin{tabular}{|l|l|l|l|}
\hline & Ecuación & $\mathbf{R}^{2}$ & Días \\
\hline Sin BPA & $\log _{10} T d=-0,012 \mathrm{x}+1,5596$ & 0,991 & 16,1 \\
\hline Con BPA & $\log _{10} \mathrm{Td}=-0,0129 \mathrm{x}+1,7671$ & 0,980 & 42,8 \\
\hline
\end{tabular}

del queso costeño. Cabe mencionar que no existen muchas investigaciones relacionadas con queso costeño y la determinación de su vida útil microbiológica. En investigaciones futuras, se considera pertinente determinar los compuestos responsables de la mencionada actividad antimicrobiana, así como realizar estudios sobre la aceptación sensorial de los quesos recubiertos y evaluar la influencia de otros tipos métodos de extracción de compuestos bioactivos, así como otras fuentes vegetales.

Conflictos de intereses: El manuscrito fue preparado y revisado con la participación de todos los autores, quienes declaramos que no existe conflicto de intereses que ponga en riesgo la validez de los resultados presentados. Financiación: Esta investigación fue llevada a cabo en la Universidad de Cartagena.

\section{REFERENCIAS}

1. ARNON, R.; POVERENOV, E. 2018. Improving food products' quality and storability by using Layer-by-Layer edible coatings. Trends in Food Science \& Technology. 75:81-92.

https://doi.org/10.1016/j.tifs.2018.03.003
2. ARROYO, C.; CEBRIÁN, G.; PAGÁN, R.; CONDÓN, S. 2011. Inactivation of Cronobacter sakazakii by ultrasonic waves under pressure in buffer and foods. Internal J. Food Microbiology. 144(3):446-454.

https://doi.org/10.1016/j.ijfoodmicro.2010.10.033

3. BARANYI, J.; ROBERTS, T. 1994. A dynamic approach to predicting bacterial growth in food. Internal J. Food Microbiol. 23(3-4):277-294.

4. BERMÚDEZ-AGUIRRE, D; BARBOSA-CÁNOVAS, G.V. 2008. Study of butter fat content in milk on the inactivation of Listeria innocua ATCC 51742 by thermo-sonication. Innovative Food Science and Emerging Technologies. $9(2): 176-185$.

https://doi.org/10.1016/j.ifset.2007.07.008

5. CANO, E.; CHÁFER, N.; CHIRALT, B.; MOLINA, P.; BORRÁS, L.; BELTRÁN, M.; GONZÁLEZ, M. 2016. Quality of goat's milk cheese as affected by coating with edible chitosan essential oil films. International J. Dairy Technology. 70(1):68-76. https://doi.org/10.1111/1471-0307.12306 
6. CASTRO-ROSAS, J.; FERREIRA-GROSSO, C.R.; GÓMEZ-ALDAPA, C.A.; RANGEL VARGAS, E.; RODRÍGUEZ-MARIN, M.L.; GUZMÁN-ORTIZ, F.A.; FALFAN-CORTES, R.N. 2017. Recent advances in microencapsulation of natural sources of antimicrobial compounds used in food - A review. Food Research Internal. 102:575-587.

http://dx.doi.org/10.1016/j.foodres.2017.09.054

7. CHANDRASEKARAN, R.; THAILAMBAL, V.G. 1990. The influence of calcium ions, acetate and L-glycerate groups on the gellan double-helix. Carbohydrate Polymers. 12:431442.

https:/ / doi.org/10.1016/0144-8617(90)90092-7

8. COSTA, M.L.; MACIEL, L.C.; TEIXEIRA, J.A.; VICENTE, A.A.; CERQUEIRA, M.A. 2018. Use of edible films and coatings in cheese preservation: Opportunities and challenges. Food Research Internal. 107:84-92. https://doi.org/10.1016/j.foodres.2018.02.013

9. DESOUSA, C.; DOS ANJOS, G.; NOBREGA, R.;MAGATON, A.; MIRANDA, F.; DIAS, F. 2020. Greener ultrasoundassisted extraction of bioactive phenolic compounds in Croton heliotropiifolius Kunth leaves. Microchemical Journal. 159:105-525.

https://doi.org/10.1016/j.microc.2020.105525

10. FRAZIER, W.; WESTHOFF, D. 2003. Microbiología de los alimentos. Editorial Acribia, S.A. Zaragoza, España. p.371401.

11. GIE, L.; OLA, L.; NAZAMID, S.; KHAIRUNNIZA, B. 2018. Effect of chitosan and carrageenan-based edible coatings on post-harvested longan (Dimocarpus longan) fruits. J. Food. 16(1):490-497.

https://doi.org/10.1080/19476337.2017.1414078

12. GONZÁlEZ, C.; CERVANTES, Y.; CARABALLO, L. 2016. Conservación de la guayaba (Psidium guajava L.) en postcosecha mediante un recubrimiento comestible binario. Temas Agrarios. 21(1):54-64.

13. GONZÁLEZ, C.; GUARDO, P.; SÁNCHEZ, C.; ALVEAR, P.; GARCÍA, Z. 2018. Effect of Schinopsis balansae, Gliricidia sepium, aqueous extract and ultrasound on viscoelastic behavior of edible film. Contemporary Engineering Sciences. 11(47):2307-2315. https://doi.org/10.12988/ces.2018.85216

14. GONZÁLEZ, R.; SALAZAR, J.; PÉREZ, J. 2013. Obtaining size-controlled microcapsules by ionic gelation with high and low acyl gellans containing Lactococcus lactis. Rev. Col. Biotecnología. 15(2):82-97.

http://dx.doi.org/10.15446/rev.colomb.biote.v15n2.35808
15. GORRASI, G.; BUGATTI, V; TAMMARO, L.; VERTUCCIO, L.; VIGLIOTTA, G.; VITTORIA, V. 2016. Active coating for storage of Mozzarella cheese packaged under. Food Control. 64:10-16.

https://doi.org/10.1016/j.foodcont.2015.12.002

16. HOOVER, G. 2000. Ultrasound. J. Food Sci. Suppement Kinetics of microbial inactivation for alternative. Food processing technologies. 65(8):93-95.

17. HUANG, H.; XU, Q.; BELWAL, T.; LI, L.; AALIM, H.; WU, Q.; DUAM, Z.; ZHANG, X.; LUO, Z. 2019. Ultrasonic impact on viscosity and extraction efficiency of polyethylene glycol: a greener approach for anthocyanins recovery from purple sweet potato. Food Chem. 283:59-67. https://doi.org/10.1016/j.foodchem.2019.01.017

18. KABORE, A.; TRAORE, A.; NIGNAN, M.; GNANDA, B.I.; BAMOGO, V.; TAMBOURA, H.H.; BELE, M.A.M.G. 2012. In vitro anthelmintic activity of Leuceana leucocephala (Lam.) De Wit. (Mimosaceae) and Gliricidia sepium (Jacq.) Kunth ex Steud (Fabaceae) leave extracts on Haemonchus contortus ova and larvae. J. Chem. Pharma. 4(1):303-309.

19. MINISTERIO DE LA SALUD, MINSALUD. 1986. Resolución número 02310 de 1986 del 24 febrero 1986 "Por la cual se reglamenta parcialmente el Título V de la Ley 09 de 1979, en lo referente a procesamiento, composición, requisitos, transporte y comercialización de los derivados lácteos.

20. NAZLI, R.; SOHAIL, T.; NAWAB, B.; YAQEEN, Z. 2011. Antimicrobial property of Gliricidia sepium plant extract Pakistan. Journal of Agricultura Res. 24(1-4):51-55.

21. OSORIO, E.; GÓMEZ, V.; OSORIO, E.; OSSA, O. 2017. Preparation of carrageenan biofilms mixed with silver nanoparticles by biological synthesis method. Dyna. 84(201):82-87.

http://dx.doi.org/10.15446/dyna.v84n201.55702

22. PATIL, S.A.; PRABHAKARA, C.; HALASANGI, B.; TORAGALMATH, S.; BADAMI, S. 2015. DNA cleavage, antibacterial, antifungal and anthelmintic studies of Co (II), $\mathrm{Ni}(\mathrm{II})$ and $\mathrm{Cu}(\mathrm{II})$ complexes of coumarin Schiff bases: synthesis and spectral approach. Spectrochim. Acta Part A Mol. Biomol. Spec. 137:641-651. https://doi.org/10.1016/j.saa.2014.08.028

23. PERALTA, M.; ODILIA, A.; HIGUERA, B.; SOTO, V.; ACEDO, F. 2015. Fabrication of an antimicrobial active packaging and its effect on the growth of pseudomonas and aerobic mesophilic bacteria in chicken. Vitae. 22(2):111120.

https://dx.doi.org/10.17533/udea.vitae.v22n2a05 
24. REZEK, J.; MASON, J.; VESNA, L.; ZORAN, H.; LJUBÍC, H. 2008. Effect of ultrasound treatment on solubility and foaming properties of whey protein suspensions. J. Food Engineering. 86(2):281-287.

https://doi.org/10.1016/j.jfoodeng.2007.10.004

25. RÍOS- DE ÁlVAREZ, L.; JACKSON, F.; GREER, A.; BARTLEY, Y.; BARTLEY, D.J.; GRANT, G.; HUNTLEY, J.F. 2012. In vitro screening of plant lectins and tropical plant extracts for anthelmintic properties. Veterinary Parasitology. 186(3-4):390-398.

https://doi.org/10.1016/j.vetpar.2011.11.004

26. RUÍZ, P.; MENCO, M.; CHAMS, C. 2017. Microbiological evaluation of artisan coastal cheese and hygieniclocative evaluation of small shops in Córdoba, Colombia. Rev. Salud Pública. 19(3):311-317.

http://dx.doi.org/10.15446/rsap.v19n3.54853

27. SANDERSON, G.R. 1990. Gellan gum. In: Harris, P. (Ed). Food gels. Elsevier Applied Science (New York). p.201-231.
28. SINHA, S.N. 2013. Phytochemical profiles and antioxidant activities of the leaf extracts of Gliricidia sepium. Internal J. Innovations in Bio-Sciences. 3(3):87-91.

29. TYLE, P. 1993. Effect of size, shape and hardness of particles in suspensión on oral texture and palatability. Acta Psychologica. 83:111-118.

30. VON SON-DE FERNEX, E.; ALONSO DÍAZ, M.A.; VALLES DE LA MORA, B.; MENDOZA DE GIVES, P.; GONZALEZ CORTAZAR, M.; ZAMILPA A. 2017. Anthelmintic effect of $2 \mathrm{H}$-chromen-2-one isolated from Gliricidia sepium against Cooperia punctata. Experimental Parasitology. 178:1-6.

https://doi.org/10.1016/j.exppara.2017.04.013

31. ZHAO, R.; SUN, J.; TORLEY, P.; WANG, D.; NIU, S. 2008. Measurement of particle diameter of Lactobacillus acidophilus microcapsule by spray drying and analysis on its microstructure. World Journal of Microbiology Biotechnology and Biotechnology. 24:1349-1354. https://doi.org/10.1007/s11274-007-9615-0 